\title{
Curved Lane Detection using Improved Hough Transform and CLAHE in a Multi-Channel ROI
}

\author{
Gurjyot Kaur \\ M Tech Student \\ Department of Computer Science \\ Guru Nanak Dev University \\ Amritsar
}

\author{
Amit Chhabra \\ Assistant Professor \\ Department of Computer Science \\ Guru Nanak Dev University \\ Amritsar
}

\begin{abstract}
With the increase in advent of intelligent vehicle technology, LDWS technique is actively developing to provide assistance to drivers in generating warning signal in case of lane departure. In this paper, the input image is divided into four regions of interest. Then the CLAHE is applied on the given ROI for better image contrast and removal of noise using gradient filter. Improved Hough Transform is applied on the given image for the detection of curved and straight lanes. After detecting the lanes, they are colored with green color so that they are clearly visible. The proposed algorithm is analysed based on the parameters like Accuracy, BER, Fmeasure, and Sensitivity. The analysis shows that this algorithm provides efficient and effective performance compared to the existing algorithm.
\end{abstract}

\section{Keywords}

ADAS, LDWS, Lane Detection, Hough Transform, ROI

\section{INTRODUCTION}

With increase in the number of road accidents, it has lead to concern over the nature of road accidents. In most cases, human error is the cause of accidents. Thus great efforts are required to aid the driver in driving process to reduce the possibility of human error.

The most significant development for intelligent vehicles is advanced driver assistance system (ADAS) [5]. It involves a camera-assisted system which takes real time images from the surroundings and displays relevant information to the driver. Various safety provisions are provided to avoid accident and collision by offering techniques that alert the driver of potential problems.

The development of LDWS (Lane Departure Warning System) technology that recognizes the lane to generate a warning signal to the lane departure is in progress actively. Although significant development has been made in these systems for detecting straight lanes, but little effort has been made for the detection of curved lanes. Also various improvements in Hough Transformation have been ignored for better lane detection.

In this paper, based on the image taken by the front camera, Improved Hough Transformation will be applied that detects and outputs the lane ahead. Firstly set the given input image into four regions of interest. Then the CLAHE is implemented on the given image to improve local contrast. After it, Gaussian filter is applied for the removal of noise. Then the Improved Hough Transform is applied for the detection of both straight and curved lanes.

\section{RELATED WORK}

Jae-Hyun Cho et al. (2014)[1] applied the Hough transform with optimized the accumulator cells in the four ROI in parallel and detects lanes with high efficiency. Although Hough Transform can detect only straight lines, the poor lane recognition rate on the curve road has been resolved fairly.

Chan Yee Low et al. (2014) [2] presented a robust road lane marker detection algorithm to detect the left and right lane markers. The algorithm consists of optimization of Canny edge detection and Hough Transform. Canny edge detection performs features recognition then followed by Hough Transform lane generation. Hough Transform is applied to find relevant lines that can be used as the left and right lane boundaries. Reducing the image to smaller region of interest can reduce high computational cost.

Dajun Ding et al. (2013) [3] proposed an algorithm based on road ROI determination for detecting road region using information of vanishing points and line segments. Unnecessary information included in input images was analyzed in a region of interest (ROI) for reducing amount of computation. Hough Transform is used for detecting line segments. Road ROI is determined automatically in every frame. This method works effectively in various road conditions.

Hongli Fani and Weihua Wang (2013) [4] proposed a new algorithm for color road image edge detection. The original color data in RGB color model were converted to Lab color model and the difference information between the gray image from $\mathrm{L}$ channel and the red-green image was obtained with different image method, and the threshold was obtained using optimal threshold value algorithm, then edge detection was carried out. The results show that algorithm has high resistance to noise and retain better edges for color road image edge detection than the traditional algorithms.

N. Phaneendra et al. (2013) [5] adopted lane detection method which consisted of image preprocessing, binary processing and dynamical threshold choosing, and Hough transform model fitting. Instead of Hough transform, Kalman filter was used for improving lane detection performance.Based on distance between lane and center of bottom in captured image coordinate, decision making of lane departure was proposed. Efficiency and feasibility of the solution was indicated by the experimental results.

Wang Jian et al. (2013) [6] found that when selected seed points are correct, the accuracy of the method of road region extraction based on regional growth is high. This method can identify lane region exactly. But when selected seed points are wrong, lane identification will fail, and lead into some interference information. In this paper, the deficiency of this 
method is improved. It uses this method to identify lane feature region, and introduces the area threshold value to filter scanned region growing area. This can reduce the interference of useless information on lane identification. Lane identification algorithm and lane departure warning algorithm achieve good experimental results from speed and recognition rate.

F. Mariut (2012) [7] proposed a simple algorithm that detects the lane marks and its characteristics and is able to determine the travelling direction. The Hough Transform was used to detect the lines in images. A technique was developed for extracting inner margin of lane to ensure right detection of lane mark.

Kamarul Ghazali et al. (2012) [8] proposed an algorithm for detecting unexpected lane changes. An algorithm based on $\mathrm{H}-$ maxima and improved Hough Tranform was proposed which defines region of interest from input image and then divides the image into near and far field of view. Hough Transform was applied on near field of view to detect lane marks after noise filtering. The results showed that this algorithm is effective for straight roads.

Yong Chen and Mingyi He (2012) [9] proposed an algorithm called lane boundaries projective model (LBPM) for sharp curved lanes. Using lane model lane posterior probability is derived and then using particle swarm optimization lane maximum posteriori probability is found. Through lane model, lane boundaries are positioned and lane geometric structure is calculated. The results show that this method is effective for sharp curved lanes. But it detects only host lane.

Zhiyuan Xu et al. (2009) [10] presented a method based on CLAHE for removing the effect of fog. A maximum value is established to clip the histogram and distribute the pixels clipped to each gray level. This method can limit the noise in an image while enhancing image contrast

\section{ALGORITHM DESCRIPTION}

Figure 1 shows flow of the algorithm proposed.

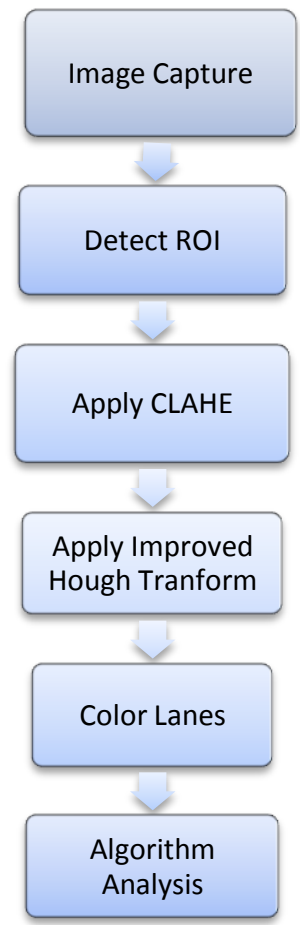

Figure 1: General flowchart of Lane Detection algorithm

\section{Step 1: Capture road image using visual sensor}

The first step of this algorithm involves capturing the road images using the front camera provided in the vehicle. The images can be taken from different viewpoints. Then these pictures are individually processed for large amount of information.

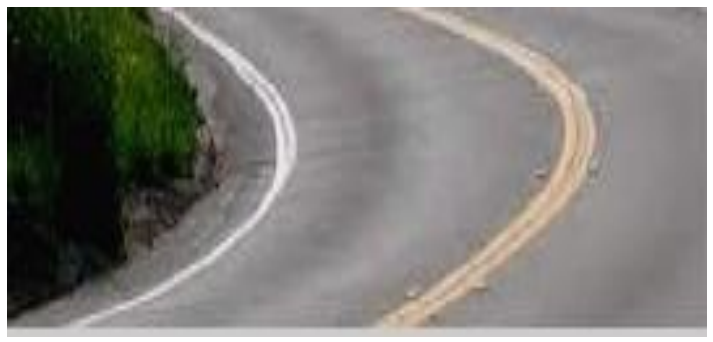

Figure 2: Original Image

Step 2: Detect ROI

After capturing the image, given image is divided [1] into four regions of interest (ROI): upperRight corner, lowerRight corner, upperLeft corner, lowerLeft corner. Select the region of interest on which the given algorithm is to be applied.

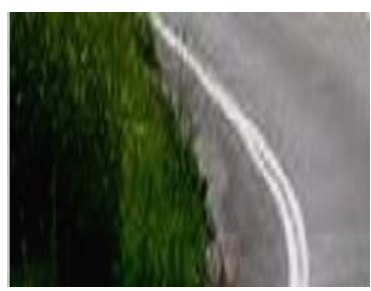

(a)

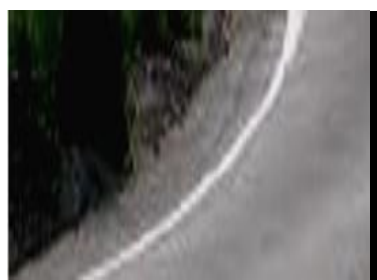

(c)

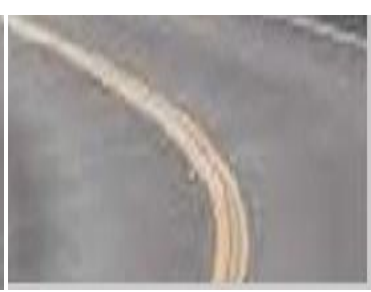

(b)

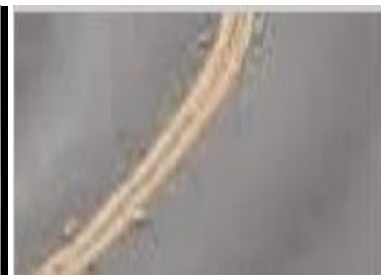

(d)
Figure 3: (a)upperLeft (b)upperRight (c)lowerLeft (d)lowerRight

Step 3: Apply CLAHE

CLAHE [10] is basically used to improve local contrast of an image more than standard histogram equalization does by bringing out more details but still has the tendency to amplify noise. After performing CLAHE, guided filter is also used on the image for the removal of noise.

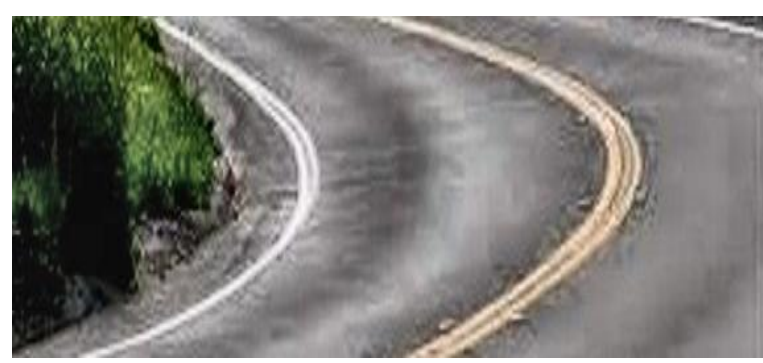

Figure 4: Image after CLAHE

Step 4: Apply Improved Hough Transform 
The Hough Transform [7] is basically used for detection of straight lanes and roads. The traditional Hough Transform can be improved for detection of curved lanes. It is popular for the detection of lanes in the presence of noise and missing data.

Through each point of image it is able to find the dominant lines by counting each unique equation for every possible line.

A line in a Hough Transform [2] is represented by $r$ and $\theta$, which are its polar co-ordinates. The $r$ parameter represents the distance between the line and the origin, and the $\theta$ parameter represents the angle of the vector from the origin to the closest point on the line.

Using this new parameterization, a line can be expressed as in (2), where $r\left[0, r_{\max }\right], \mathrm{r}_{\max }$ is a chosen value, and $\theta[0,2 \Pi]$.

$$
\left.\mathrm{y}=\frac{-x \cos (\theta)}{\sin (\theta)}+r \sin \theta\right)
$$

This relation can be rearranged into

$$
r=x \cos (\theta)+y \sin (\theta)
$$

This way, a point $(x, y)$ from the image plane, can be mapped into a curve in $(r, \theta)$ space.

The Hough Transform on the given input image is shown in Figure

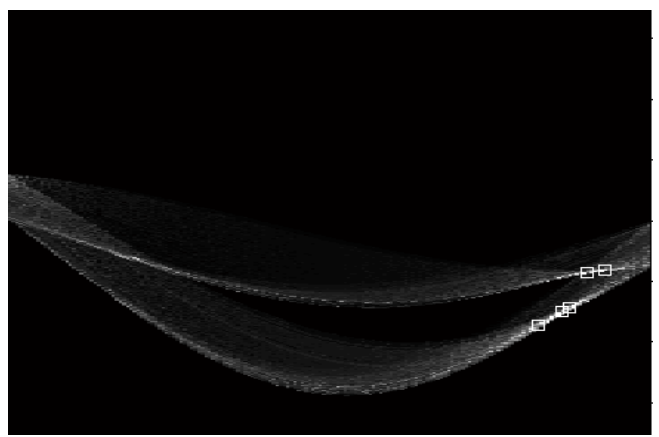

Figure 5: Hough results

Step 5: Color Lanes

After detecting the curved and straight lanes using Improved Hough Transform, those lanes are colored green so that they are clearly visible and user is warned of lane departure.

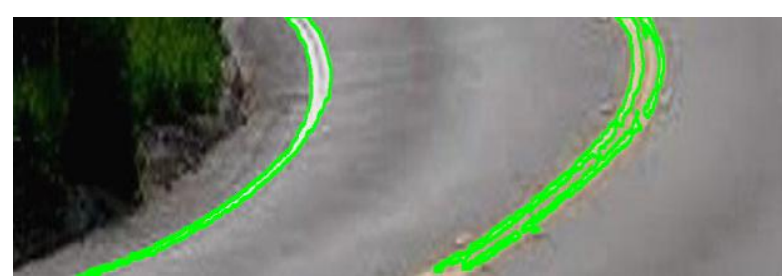

Figure 6: Colored Lanes

Step 6: Algorithm Analysis

The results of the given algorithm are analyzed on the basis of parameters like accuracy, sensitivity, BER and F-measure.

\section{EXPERIMENTAL RESULTS}

This section presents the performance of proposed algorithm based on parameters like accuracy, sensitivity, BER, Fmeasure. The algorithm manages to detect both the straight and curved lanes. The proposed algorithm is implemented on images captured from the front camera in the vehicle.
Approximately 10 images are used to validate the algorithm and for reliable verification same algorithm is applied on both straight and curved lanes.

\subsection{Accuracy}

Accuracy is the measure of the total number of predictions that were correct.

$$
\text { Accuracy }=\frac{\text { True Positive }+ \text { True Negative }}{\text { Total Population }}
$$

Higher value of accuracy means that more number of predictions was accurate. The chart given in Figure 7 shows the increased accuracy of proposed algorithm over existing algorithm.

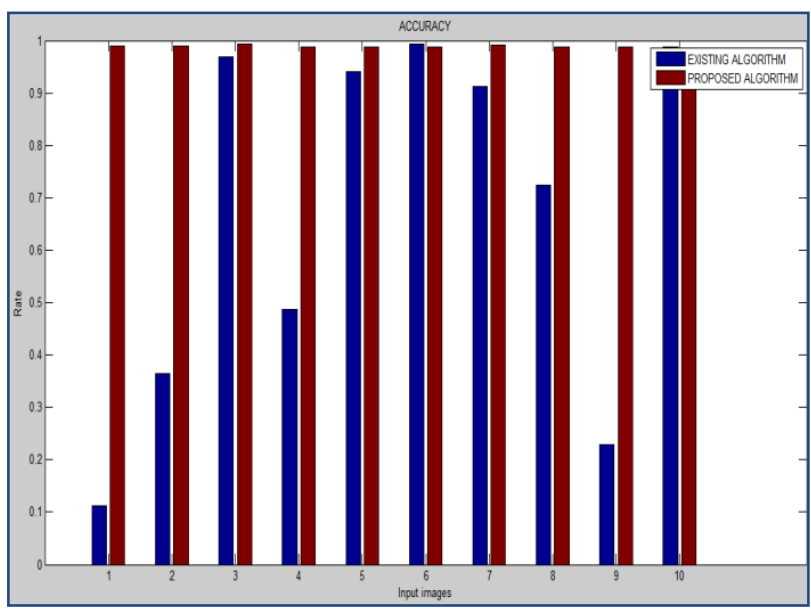

Figure 7: Accuracy Analysis

\subsection{F-measure}

F-measure is the measure of a test's accuracy. It considers both precision and recall to compute the score. F-measure is the weighted averaged of precision and recall. Higher value of F-measure means higher quality.

$$
\text { F-measure }=\frac{\text { precision } * \text { recall }}{\text { precision }+ \text { recall }}
$$

The chart given in Figure 8 shows increased F-measure value of proposed algorithm over existing algorithm. Thus proposed algorithm is more accurate.

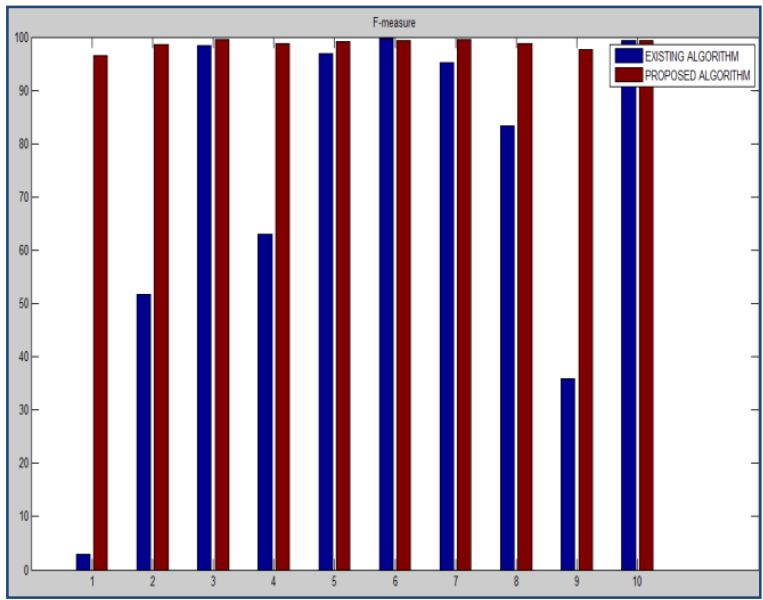

Figure 8: F-measure analysis 


\subsection{Sensitivity}

Sensitivity or recall is the proportion of positive cases that were accurately identified. Higher value indicates more number of positive cases.

$$
\text { Sensitivity }=\frac{\text { True Positive }}{\text { Total Numberof Positive Cases }}
$$

Chart given in Figure 9 shows the increased value of sensitivity for proposed algorithm as compared to existing algorithm.

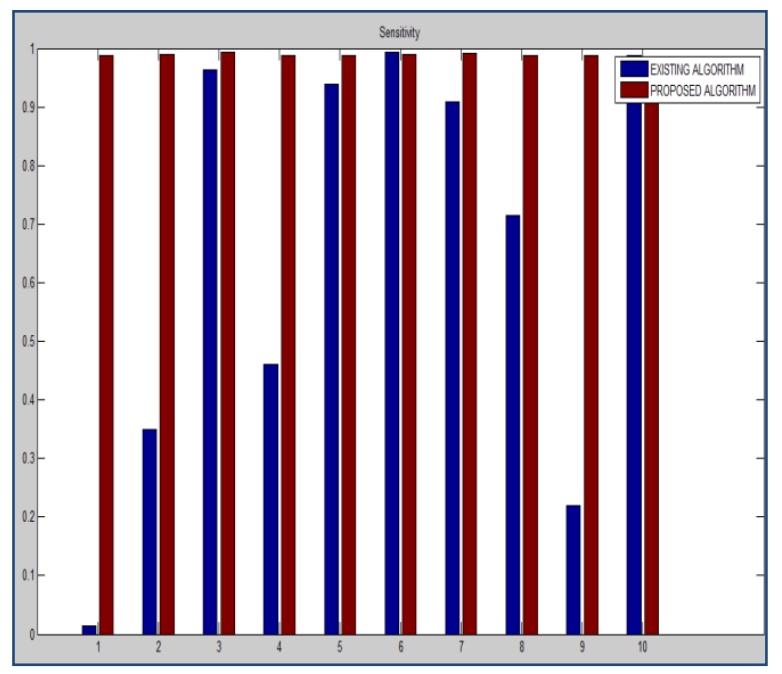

Figure 9: Sensitivity analysis

\subsection{BER}

Bit Error Rate (BER) is the number of bit errors divided by the total number of bits transferred during a specific interval of time. It is basically used to test the quality of an image.

Figure 10 shows the BER Analysis of existing and proposed algorithm. Value of BER is negligible for the proposed algorithm.

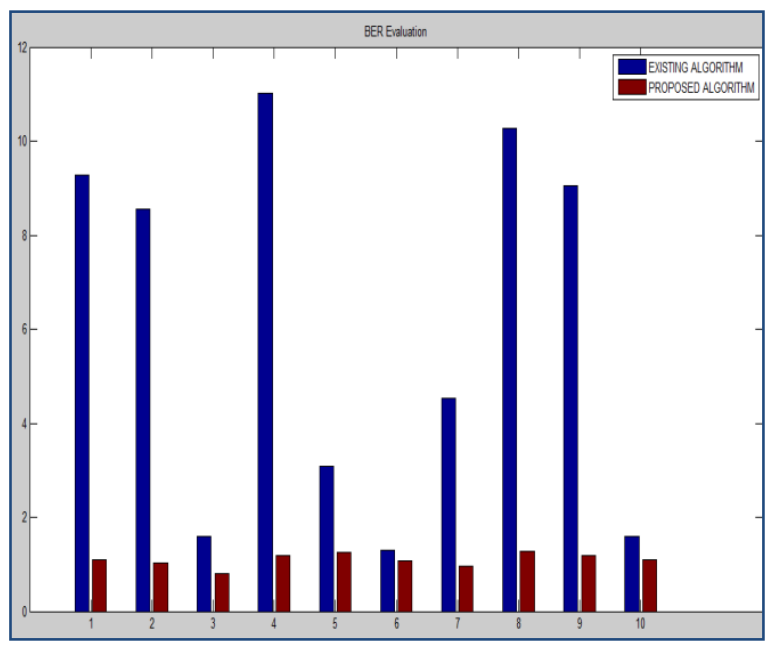

Figure 10: BER Analysis

\section{CONCLUSIONS}

In this paper, the given input image is divided into four ROI and a preprocessor CLAHE is applied for better image contrast. Then the Improved Hough Transform is applied for the detection of curved and straight lanes. After detecting the lanes, they are colored green.

The results show that the proposed algorithm is effective in detecting both straight and curved lanes. Four parameters i.e Accuracy, BER, Sensitivity and F-measure are used for the analysis of proposed algorithm. These parameters showed that the proposed algorithm is efficient and effective as compared to existing algorithm.

\section{REFERENCES}

[1] Cho, Jae-Hyun, Young-Min Jang, and Sang-Bock Cho. "Lane recognition algorithm using the Hough transform with applied accumulator cells in multi-channel ROI." In Consumer Electronics (ISCE 2014), The 18th IEEE International Symposium on, pp. 1-3. IEEE, 2014.

[2] Low, Chan Yee, Hairi Zamzuri, and Saiful Amri Mazlan. "Simple Robust Road Lane Detection Algorithm". IEEE, 2014.

[3] Ding, Dajun, Chanho Lee, and Kwang-yeob Lee. "An adaptive road ROI determination algorithm for lane detection." In TENCON 2013-2013 IEEE Region 10 Conference (31194), pp. 1-4. IEEE, 2013.

[4] Fani, Hongli, and Weihua Wang. "Edge Detection of Color Road Image Based on Lab Model." In Computational and Information Sciences (ICCIS), 2013 Fifth International Conference on, pp. 298-301. IEEE, 2013.

[5] Nalla, Phaneendra, GCL Abhiraman Goud, and V. Padmaja. "ACCIDENT AVOIDING SYSTEM USING LANE DETECTION." IJRECE 1, no. 1 (2013): 01-04.

[6] Jian, Wang, Sun Sisi, Gong Jingchao, and Cao Yu. "Research of lane detection and recognition technology based on morphology feature." In Control and Decision Conference (CCDC), 2013 25th Chinese, pp. 3827-3831. IEEE, 2013.

[7] Mariut, F., C. Fosalau, and D. Petrisor. "Lane mark detection using Hough Transform" In Electrical and Power Engineering (EPE), 2012 International Conference and Exposition on, pp. 871-875. IEEE, 2012.

[8] Ghazali, Kamarul, Rui Xiao, and Jie Ma. "Road lane detection using H-maxima and improved hough transform." In Computational Intelligence, Modelling and Simulation (CIMSiM), 2012 Fourth International Conference on, pp. 205-208. IEEE, 2012.

[9] Chen, Yong, and Mingyi He. "Sharp curve lane boundaries projective model and detection." In Industrial Informatics (INDIN), 2012 10th IEEE International Conference on, pp. 1188-1193. IEEE, 2012.

[10] Xu, Zhiyuan, Xiaoming Liu, and Na Ji. "Fog removal from color images using contrast limited adaptive histogram equalization." In Image and Signal Processing, 2009. 